Draft version February 26, 2018

Preprint typeset using $\mathrm{LATEX}_{\mathrm{E}}$ style emulateapj v. 12/16/11

\title{
XMM-NEWTON SURVEY OF LOCAL O VII ABSORPTION LINES IN THE SPECTRA OF GALACTIC X-RAY SOURCES
}

\author{
YANG LUO ${ }^{1,2,3}$, TAOtaO FAnG ${ }^{1}$, Renyi MA ${ }^{1}$ \\ Draft version February 26, 2018
}

\begin{abstract}
The detection of highly ionized metal absorption lines in the X-ray spectra of the Galactic X-ray binaries (XRBs) implies the distribution of hot gas along the sightline toward the background sources. However, the origin of this hot gas is still unclear: it can arise in the hot interstellar medium (ISM), or is intrinsic to the XRBs. In this paper, we present an XMM-Newton survey of the O VII absorption lines in the spectra of Galactic XRBs. A total of 33 XRBs were selected, with 29 low mass XRBs and 4 high mass XRBs. At more than $3 \sigma$ threshold, O VII absorption line was detected in 16 targets, among which 4 were newly discovered in this work. The average line equivalent width (EW) is centered around $\sim 20 \mathrm{~mA}$. Additionally we do not find strong correlations between the O VII EWs and the Galactic neutral absorption $\mathrm{N}_{\mathrm{H}}$, the Galactic coordinates or the distance of background targets. Such non-correlation may suggest the contamination of the circumstellar material, or the lack of constrains on the line Doppler-b parameter. We also find regardless the direction of the XRBs, the O VII absorption lines were always detected when the flux of the background XRBs reach a certain level, suggesting a uniform distribution of this hot gas. We estimate a ratio of $0.004-0.4$ between hot and neutral phase of the interstellar medium. This is the second paper in the series following Fang et al. (2015), in which we focused on the local O VII absorption lines detected in the background AGN spectra. Detailed modeling of the hot ISM distribution will be investigated in a future paper.
\end{abstract}

Keywords: galaxies: ISM — X-rays: ISM — ISM: general

\section{INTRODUCTION}

The presence of hot interstellar medium (ISM) has been predicted for a long time (for a review, see Spitzer 1956; Ferriere 2001; Putman et al. 2012). This hot gas $\left(\sim 10^{6} \mathrm{~K}\right)$ could be a result of stellar feedback (supernova explosions or stellar wind from massive stars) or shock-heated intergalactic medium (IGM) accreting to the Galactic center, therefore study of the hot ISM could have a profound implication on the theory of galaxy formation and evolution.

Current understanding of the Galactic hot gas initially comes from various broad-band observations of the diffuse soft X-ray background. At the soft Xray band, other than the extragalactic component, the solar wind charge exchange and the local hot bubble component, a contribution of the background is expected from a large-scale hot plasma present in the Galactic halo (e.g., Lallement 2004; Cappelluti et al. 2012; Henlev \& Shelton 2013; Galeazzi et al. 2014). Insights on the properties of the hot ISM also come from the study of $\mathrm{z}=0$, metal absorption lines in extragalactic active galactic nuclei (AGN). The detection of $\mathrm{z}=0$, highly ionized absorption lines (e.g, $\mathrm{Ne}$ IX, O VII and O VIII) toward bright extragalactic sources (e.g., Nicastro et al. 2002; Fang et al. 2003; Rasmussen et al. 2003) and their Galactic origin

\footnotetext{
${ }^{1}$ Department of Astronomy and Institute of Theoretical Physics and Astrophysics, Xiamen University, Xiamen, Fujian 361005, China; fangt@xmu.edu.cn

2 Theoretical Astrophysics, Department of Earth \& Space Science, Osaka University, 1-1 Machikaneyama, Toyonaka, Osaka 5600043, Japan

${ }^{3}$ Department of Physics \& Astronomy, University of Kentucky, Lexington, KY 40506-0055, USA
}

(Fang et al. 2006; Bregman \& Llovd-Davies 2007) also suggested the existence of the hot ISM.

However, current data cannot give a precise description of the spatial distribution of the hot gas. Evidence from the disk-morphology of the X-ray emission of nearby galaxies is suggestive of the gas to be confined in the disk (Tüllmann et al. 2006; Li \& Wang 2013). However, semi-analytic calculations and numerical simulations for disk galaxy formation predict the existence of extended hot gaseous halos around massive spiral galaxies due to the accretion of the intergalactic medium (e.g., White \& Frenk 1991; Mo \& Mao 2002; Maller \& Bullock 2004; Fukugita \& Peebles 2006; Crain et al. 2010; Ntormousi \& Sommer-Larsen 2010; Marinacci et al. 2011; Stinson et al. 2012; Feldmann et al.|2013; Fang et al. 2013; Sokołowska et al. 2016). The hot ISM may extend out to at least the Magellanic System, as suggested by the pressure confinement of high-velocity cloud or satellite galaxies stripping stream (Sembach et al. 2003; Collins et al. 2005; Grcevich \& Putman 2009; Lehner et al. 2012; Gatto et al. 2013). Detections of diffuse X-ray halos around massive normal spiral galaxies also suggested the existence of hot, X-ray emitting gas beyond the disk (Anderson \& Bregman 2011; Dai et al. 2012; Bogdan et al. 2012, 2013; Hodges-Kluck \& Bregman 2013; Anderson et al. 2016).

In the past few years, it has become well established that a large amount of hot gas is present along the sightline toward background XRBs. Located in the Galactic disk, the XRBs provide a potential probe of the ISM in the disk. The detection of X-ray absorption lines of highly ionized species (e.g, Ne IX, O VII and O VIII) toward the XRBs leads to the assumption that the hot gas 
is located in the Galactic thick disk (e.g., Futamoto et al. 2004; Yao \& Wang 2005; Wang et al. 2005; Yao et al. 2009; Pinto et al. 2013). This hot ISM derived from XRBs observations has an exponential scale height of several kpc (Yao \& Wang 2005; Yao et al. 2009). The hot gas near the Galactic plane may has density around $1 \times 10^{-3} \mathrm{~cm}^{-3}$ Yao et al. 2009; Hagihara et al. 2010; Sakai et al. 2014) and the temperature around $2 \times 10^{6}$ $\mathrm{K}$ (Yao \& Wang 2007). By comparing the amount of absorption detected along the sightline towards the XRBs vs. those detected toward the distant AGNs, it has been concluded that most of the hot gas is constrained within the disk (Yao et al. 2008).

However, the circumstellar medium local to XRB could also produced such absorption lines. Miller et al. (2004) analyzed the absorption lines from GX 339-4 and XTE J1650-500 and they find these highly ionized absorption lines could not be produced in the ISM, but in a photo-ionized warm absorber intrinsically. Later work on the time variability of the absorption lines show that in some targets the line strength varies between observations taken at different time (Cackett et al. 2008; Luo \& Fang 2014; Petrucci et al. 2014). These lines are likely photo-ionized by the XRB luminosity with an ionization parameter $\log (\xi)$ in the range of $1-3$ (Cackett et al. 2008; Luo \& Fang 2014). Some of the highly-ionized lines also are found to be blue-shifted, and could be modeled with outflowing photoionized gas near the accretion disk or its corona (e.g, SAX J1808.4-3658 observed by Pinto et al. 2014). Study of the origin of these highly ionized absorption lines would benefit our understanding of both the circumstellar medium intrinsic to the XRB and the ISM, as well as the hot gas content in our Galaxy in general.

In order to fully understand the origin of the absorption lines detected in the X-ray spectra of the XRBs and thereafter the properties of the hot ISM, a large sample of targets with detection of hot gas produced absorption lines is required. In this work, we perform a comprehensive analysis of the highly ionized absorption lines in the XRBs, with a focus on the highly ionized O VII absorption line at $21.6 \AA$. We have performed a comprehensive analysis of all the available data in the XMM-Newton archive with a concentration on O VII lines. Recent work by Fang et al. (2015) provided an all-sky survey of O VII toward background active galactic nuclei (AGN) from archival XMM-Newton observations, and they find a $100 \%$ sky covering fraction of O VII lines, which suggest a uniform distribution of the absorbers. Here we present the second paper in this series.

The paper is organized as follows. In section 2 , we first describe the selection of our sample and the procedure of data reduction, and then we analyze the absorption lines and present our main results in this section. We discuss the distribution of the absorption line in section 3, as well as the origins of these lines. We also comment on several targets with newly detected, O VII lines in this section. The last section is the summary.

\section{OBSERVATION AND DATA ANALYSIS}

\subsection{Target Selection and Data Reduction}

Numerous X-ray absorption lines were detected in the $\mathrm{X}$-ray spectra of the XRBs. These absorption lines sample the ISM in a variety of temperature ranges, providing rich information of the multi-phase distribution of the ISM (e.g., Ferriere 2001; Yao \& Wang 2005; Costantini et al. 2012; Pinto et al. 2013). Here we focus on highly ionized, He-like O VII for the following reasons. First, as one of the most abundance elements in universe, oxygen is a very important tracer of the metals in the ISM. Secondly, under collisional ionization, the peak temperature of O VII ionization fraction is in the range of $10^{5.5}-10^{6.5} \mathrm{~K}$, providing an effective way to probe the hot, ionized ISM. Therefore, in our work, we focused on the absorption line from $\mathrm{O}$ VII $\mathrm{K}_{\alpha}$ transition with a rest-frame wavelength of $\lambda_{\text {rest }}=21.6019 \AA$ (Verner et al. 1996; Yao et al. 2009; Liao et al. 2013).

Currently, there are three grating-based, high resolution X-ray spectrometers suitable to our study of highly ionized metal absorption lines, namely the Low and High Energy Transmission Grating Spectrometer ${ }^{4}$ (LETG and HETG) onboard the Chandra X-ray Observatory and the Reflection Grating Spectrometer ${ }^{5}$ (RGS) onboard XMMNewton X-ray telescope. RGS has relatively high collecting area than both LETG and HETG. For RGS, there are two units, RGS1 and RGS2. However, one CCD assembly in each RGS unit has an operation failure due to electronics problems. These are RGS1 CCD7 and RGS2 CCD4, roughly covering the wavelength ranges 11-14 $\AA$ and 20-24 $\AA$ respectively, in first order. Therefore, in this work, we will focus on the RGS1 data only.

Based on Chandra observation, Luo \& Fang (2014) has already studied a sample of Ne IX line toward XRBs. Due to the small effective area and strong galactic absorption, the detected O VII photon counts from Chandra are small. In our selection of all XRBs observations, the number of XRBs observed by Chandra is smaller than that of RGS, therefore we could not make a large well-defined O VII sample from Chandra. At wavelength of $19.0 \AA$ the RGS1 has one instrumental feature which make it unlikely for a good fit of $\mathrm{O}$ VIII $\mathrm{K}_{\alpha}$ line. At wavelength of $18.6 \AA$ we found some weak detections of $\mathrm{O}$ VII $\mathrm{K}_{\beta}$ line in part of our sample. Due to the small line oscillation strength, the line EWs and their significances could not be well constrained. We have tried to jointly fit the $\mathrm{O}$ VII $\mathrm{K}_{\alpha}$ and $\mathrm{K}_{\beta}$, and the joint-fit could not give a better improvement of the line EWs. Therefor in our work, we will concentrate on the $\mathrm{O}$ VII $\mathrm{K}_{\alpha}$ line from RGS1 observation.

We have compiled a list of XRBs from the XMMNewton archive. We inspected the RGS data of these targets and selected the ones with relatively high photon statistics. We define our selection criteria as the counts per resolution element (CPRE) at wavelength near O VII must be at least 20 photos. For each source, we define the CPRE, which is the photon counts within a bin size of the spectral resolution $(50 \mathrm{~m} \AA)$ :

$$
C P R E=F_{\lambda} A T \Delta \lambda
$$

where $F_{\lambda}$ is the photon flux, $A$ is the effective area of the detector, $T$ is the observation time, and $\Delta \lambda$ is the width

\footnotetext{
4 see Chandra Proposers' Observatory Guide at:

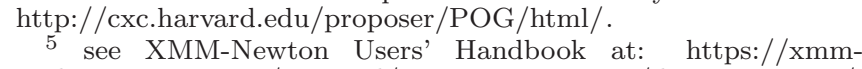
tools.cosmos.esa.int/external/xmm_user_support/documentation/uhb/.
} 


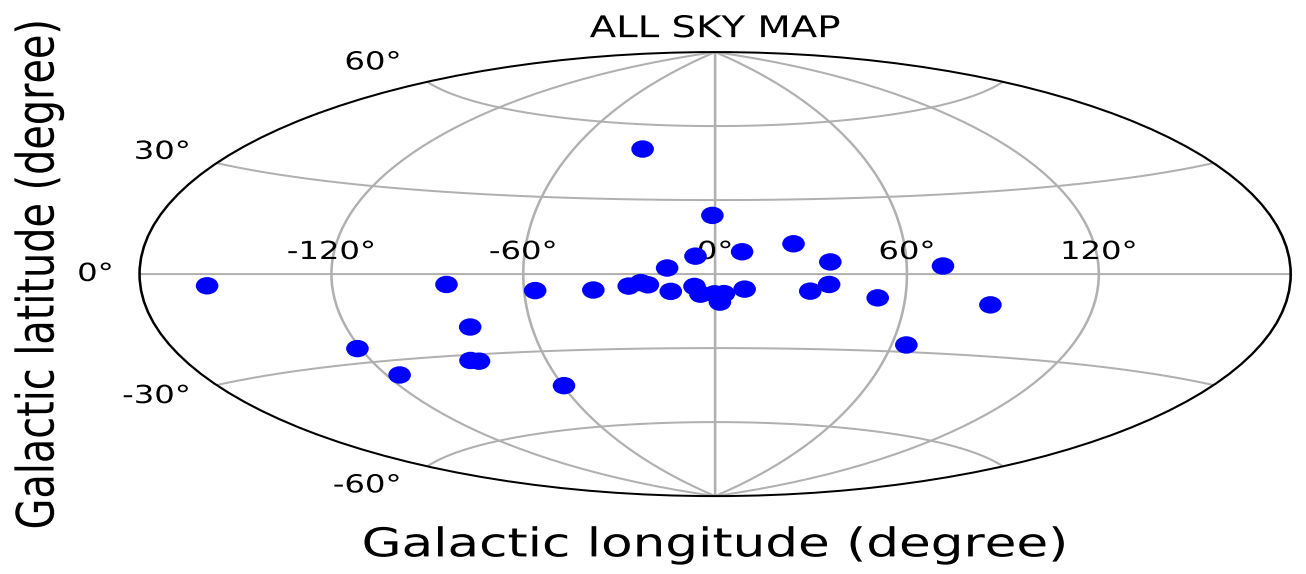

Figure 1. All-sky Hammer-Aitoff projection of targets in our sample.

of one resolution element. We also ignore those targets with O VII K-shell emission lines (consisting of resonance, inter-combination and forbidden lines at 21.60, 21.80 and $22.10 \AA$, respectively) to avoid contamination. This leads to a sample of 33 sources, with 29 low mass $\mathrm{X}$-ray binaries (LMXBs) and 4 high mass X-ray binaries (HMXBs). In Figure 1 we plot the all-sky HammerAitoff projection of our targets in the Galactic coordinates. Most of our targets are located in or near the Galactic disk.

We have reduced the spectra with the Science Analysis System (SAS) version $13.0{ }^{6}$. RGS data are processed with the SAS task rgsproc. Events with flags of $B A D \_S H A P E$, $O N_{-} B A D P I X, \quad O N_{-} W I N D O W_{-} B O R A D E R$, and $B E L O W \_A C C E P T A N C E$ are all rejected. We also did not keep the cool pixels (keepcool=no). We produced the light curves for the background in CCD9 following the XMM-SAS guide in order to remove time intervals that are contaminated with soft proton flares and spurious events. For each observation, only the first order spectrum was extracted.

\subsection{Absorption Line Analysis}

Many of these sources were observed multiple times. To avoid unreal artifacts that may be introduced in the co-added exposures, for each target we simultaneously fitted all the exposures, each with its own response. Our analysis focuses on the segment spectrum around $20-22 \AA$ and models the spectra continuum with the Galactic neutral absorption plus a power-law model, using XSPEC ver 12.7 (Arnaud 1996). For each target, while we have adopted different continuum for each exposure, the parameters of the interstellar absorption were always tied together. We also excluded wavelength regions of known detector features. Absorption lines were fitted with a Voigt line profile model developed in Buote et al. (2009). This line model has three free parameters: Column density, Doppler-b parameter, and the velocity shift of the central wavelength. We refer the reader to Buote et al. (2009) and Fang et al. (2010) for details. Since we are interested in the absorption lines produced by the local hot gas, therefore we limited the line shift-velocity within $500 \mathrm{~km} \mathrm{~s}^{-1}$. The Doppler-b pa-

\footnotetext{
${ }^{6}$ see Cookbook at: http://heasarc.gsfc.nasa.gov/docs/xmm/abc/
}

rameter is also limited in the range of 20 to $300 \mathrm{~km} \mathrm{~s}^{-1}$. The lowest temperature that can still produce a significant fraction of $\mathrm{O}$ VII is around $5 \times 10^{5} \mathrm{~K}$ under collisional ionization equilibrium, this corresponds to a thermal velocity of $\sim 20 \mathrm{~km} \mathrm{~s}^{-1}$. The upper limit is adopted by assuming the absorbing gas shall not escape from our Galaxy. For a better constraint of the line EWs, we estimate the statistical significance of the lines using Monte Carlo simulations. Briefly, we make a simulated spectra based on our fitted absorption line models and run 1,000 simulations on each targets. We fit the simulated spectra, record the measured EWs, and finally obtain the line significance (more details see Fang et al. 2010). We performed the fit by minimizing the $\mathrm{C}$-statistic, which yields less biased best-fitting parameters.

The basic information of our sample and the fitted line properties are listed in Table 4 . We show the source name and type in column 1 and 2, respectively. Column 3 is the galactic neutral hydrogen column density adopted from Kalberla et al. (2005). Columns 4, 5, 6 are the galactic latitude, longitude and distance, respectively. All of the distance are adopted from literatures. We list the total exposure time and CPRE for each source in column 7 and 8, respectively. Column 9, 10, 11 are the O VII column density, velocity shift of the line center and the Doppler-b parameter, respectively. The line equivalent width (EW) and $1 \sigma$ statistical uncertainty are given in column 12 . We show the line significance in column 13 and $C$-statistic and degree of freedom in column 14. The targets are listed in descending order based on their CPRE, which indicates of the the quality of the spectra. For targets we cannot constrain either the Doppler-b parameter or the shift-velocity of the line center, we set the velocity shift of the line center at $0 \mathrm{~km} \mathrm{~s}^{-1}$ or the Doppler-b parameter at $300 \mathrm{~km} \mathrm{~s}^{-1}$. In our sample, we find one target having detected absorption line with shift velocity larger than $500 \mathrm{~km} \mathrm{~s}^{-1}$. Such a large shift suggests most likely the absorption line is produced by the circumstellar medium local to the source. We also list this high velocity line in the table, plus with the $3 \sigma$ upper limits of $\mathrm{EW}$ for their lines at $0 \mathrm{~km} \mathrm{~s}^{-1}$. We adopt $1 \sigma$ errors throughout the paper unless otherwise mentioned.

Of the total of 33 targets, 16 show an O VII detection significance larger than $3 \sigma$ threshold, 9 are at the $2-3 \sigma$ level. Details about the detections at different significance level are listed in Table 1. Only one target has a 
Table 1

Target Detection

\begin{tabular}{lllll}
\hline \hline & $>3 \sigma$ & $>2 \sigma$ & $>1 \sigma$ & Total \\
\hline All & 16 & 25 & 32 & 33 \\
LMXB & 14 & 22 & 28 & 29 \\
HMXB & 2 & 3 & 4 & 4 \\
\hline
\end{tabular}

Note. - Target detection in our line measurements. Columns 2, 3, 4 list the targets detected with a significance larger than $3,2,1 \sigma$, respectively. The last column is the total numbers of targets in each group.

weak absorption line presented as significance less than $1 \sigma$. The difference between HMXB and LMXB mostly comes from the mass of the companion star and the way accretion of matter occurs. The spectra of HMXB is sensitive to the properties of the companion wind. In our sample, we have four HMXBs, two (LMC X-3 and Cygnus $\mathrm{X}-1$ ) of which show $\mathrm{O}$ VII detection significance larger than $3 \sigma$. $4 \mathrm{U}$ 0513-40 has shift-velocity larger than $500 \mathrm{~km} \mathrm{~s}^{-1}$, and its detection significance is $2.9 \sigma$. In the spectrum of EXO 0748-676, O VII Kshell emission is clearly shown near $21.8 \AA$ Cottam et al. 2002; van Peet et al. 2009), whereas one absorption line at $21.6 \AA$ is also detected here with a significance of $4.1 \sigma$. Of the targets with significance larger than $3 \sigma, 4$ targets are newly newly detections: Sco X-1, Swift J1753.50127, MAXI J0556-332 and MAXI J1910-057.

For a more careful examination of the line significances, we have tested the detections by another method of Monte Carlo simulations. We first fit and simulate the continuum spectrum, and then we fit the simulated data with a continuum model and record the C-statistic. We add the absorption model, and record the change of the C-statistic. We have performed 10,000 simulations for three test targets to check how many times the changed C-statistic given by adding a O VII line provides a result better than the measurement. These three test targets, Sco X-1, EXO 0748-676 and GS 1826-238 are selected due to their different statistics and we expect this small sample will provide a very interesting view on the overall significance of the total sample. We get the line significances for these three targets are $>6 \sigma, 2.8 \sigma$, and $3.6 \sigma$, respectively. These results are in general consistent with the significances we have obtained in the first Monte Carlo simulations.

In Figure 2, we show the histogram distribution of the $\mathrm{O}$ VII $\mathrm{K}_{\alpha}$ line EW for targets detected at $>1 \sigma$ significance. The measured line EWs are centered around $\sim$ $20 \mathrm{m \AA}$, with a range of $10-40 \mathrm{m \AA}$. In Figure 3, we show the line detection rate, or the sky covering fraction, as a function of the photon counts CPRE. The solid, dashed, and dotted lines are for the detections with at least 1, 2, and $3 \sigma$ significance, respectively. For all cases, the detection fractions increase steadily as the increasing CPRE. For targets with CPRE more than about 400 counts the $3 \sigma$ detection fraction reaches $100 \%$. Such detection rate implies a uniform distribution of the hot gas in all directions.

Some of the O VII detections were reported previously. We have compared our results with previous measurements. We list our work and previous studies in Table 2

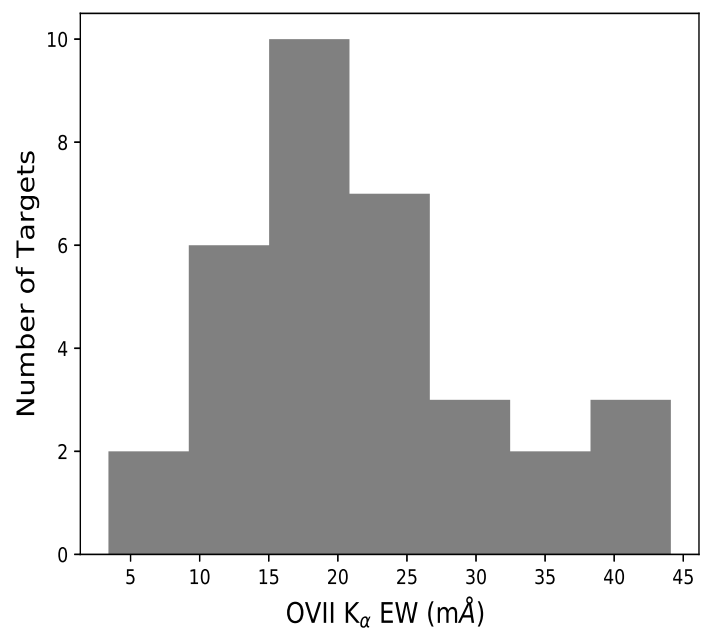

Figure 2. Histogram distribution of the O VII $\mathrm{K}_{\alpha}$ line EW at detected significance larger than $1 \sigma$. The targets have a EW centered around $20 \mathrm{~m} \AA$ and distribute in the range of $10-40 \mathrm{~m} \AA$.

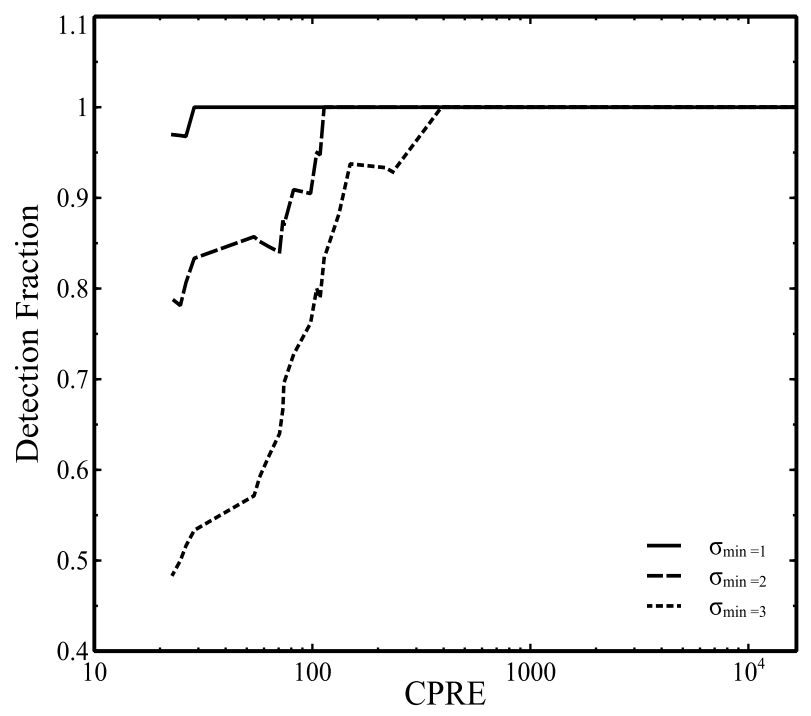

Figure 3. Detection fraction as a function of source CPRE. The fractions for absorption lines detected with at least 1,2 , and $3 \sigma$ significance are presented as the solid, dashed, and dotted lines, respectively.

Column 2 shows the line EWs in our work, and columns 3 and 4 are previous results obtained with Chandra and XMM-Newton, respectively. Some of the previous results were initially listed in units of column density. For consistency, we have converted those results to units of $\mathrm{m} \AA$ with $1 \sigma$ error bar, assuming a Doppler-b parameter of $100 \mathrm{~km} \mathrm{~s}^{-1}$. Our measurements are largely consistent with previous ones within the error range. Cygnus $\mathrm{X}-2$ is a unique one because the $\mathrm{O}$ VII $\mathrm{K} \alpha$ line is not detected in Chandra. We refer the reader to Cabot et al. (2013) for a detailed analysis of the O VII line in Cygnus X2. For XTE J1817-330, Sala et al. (2007) obtained an O VII EW of $0.7 \mathrm{eV}(26.4 \mathrm{m \AA})$ and is consistent with our measurements, whereas the Chandra observation by Gatuzz et al. (2013) gives a higher value. Similar discrepancy was also presented in 4U 1820-30. One explanation for the discrepancy may be the time variability of 
Table 2

Comparison with Previous Measurements

\begin{tabular}{llll}
\hline \hline Name & $\begin{array}{l}\text { This Work } \\
(\mathrm{m} \AA)\end{array}$ & $\begin{array}{l}\text { Chandra } \\
(\mathrm{m} \AA)\end{array}$ & $\begin{array}{l}\text { XMM-Newton } \\
(\mathrm{m} \AA)\end{array}$ \\
\hline Cygnus X-2 & $18.3 \pm 1.5$ & $<6.3[1]$ & $19.6 \pm 1.3[2]$ \\
XTE J1817-330 & $34.2 \pm 5.4$ & $54.0 \pm 4.0[4]$ & $26.4 \pm 7.6[5]$ \\
4U 1820-30 & $20.5 \pm 2.1$ & $40 \pm 7.9[6]$ & $23.3 \pm 2.5[7]$ \\
& & $44.8 \pm 10.8[3]$ & $23.9 \pm 3.6[8]$ \\
GX 339-4 & $42.5 \pm 3.5$ & & $23.7 \pm 5.9[9]$ \\
SAX J1808.4-3658 & $18.5 \pm 2.5$ & & $31.5 \pm 7.2[9]$ \\
LMC X-3 & $20.5 \pm 3.1$ & $20 \pm 6[10]$ & $21.0 \pm 5.0[8]$ \\
4U 1636-54 & $27.7 \pm 4.5$ & & $19.9 \pm 7.0[9]$ \\
4U 1728-16 & $10.1 \pm 4.7$ & & $8.6 \pm 4.3[9]$ \\
4U 1254-690 & $17.6 \pm 5.3$ & & $15.2 \pm 7.6[9]$ \\
Aql X-1 & $25.0 \pm 8.6$ & & $18.1 \pm 7.2[9]$ \\
4U 1957+11 & $16.1 \pm 6.3$ & $18 \pm 18[11]$ & $19.0 \pm 19.0[11]$ \\
& & $18.7 \pm 10.4[12]$ & $18.1 \pm 7.2[9]$ \\
GS 1826-238 & $31.6 \pm 10.0$ & & $19.9 \pm 10.0[9]$ \\
4U 1735-44 & $26.4 \pm 11.3$ & & $24.7 \pm 9.7[8]$ \\
& & & $21.0 \pm 4.4[9]$ \\
\hline
\end{tabular}

Note. - [1] Yao et al. $(2009)[2 \longdiv { \text { Cabot et al. } \sqrt { 2 0 1 3 } ) \text { [3] Futamoto et al } }$ (2004) [4 Gatuzz et al. (2013) [5]Sala et al. (2007) [6]Yao \& Wang (2006) [7]Costantini et al. (2012) [8]Miller \& Bregman (2013) [9]Pinto et al. (2013) [10 Wang et al. (2005) [11 Nowak et al. (2008) [12 Yao et al. (2008)

O VII line since Chandra observations were performed in a different time. Part of our sample have been analyzed by Pinto et al. (2013). Our measurements are in general consistent with theirs. One exception is GX 339-4, for which we found a much higher EW. The difference may be caused the different data reduction procedure. For this target, its all observations were not co-added together as previous did but were simultaneously analyzed. Highly ionized absorption line in LMC X-3 has been systematically studied by Wang et al. (2005) using high resolution spectrometers on board Chandra Xray telescope, and our results are consistent with their measurements. In the Chandra spectra of Cygnus X1, Schulz et al. (2002) find an a weak detection at 21.43 $\AA$ and they identify this line as an blue-shift O VII $\mathrm{K} \alpha$ line, while the blueshifted velocity is detected differently as $2350 \mathrm{~km} \mathrm{~s}^{-1}$ and we get the velocity as $494 \mathrm{~km} \mathrm{~s}^{-1}$. This could be a signature of line variation.

\section{DISCUSSION}

\subsection{Study of ISM Distribution}

The distribution of the metal absorption lines in the spectra of different XRBs allows us to quantify how the intervening hot gas distributed in the circumstellar medium and the ISM. Here we show the dependance of the line EW on the target direction, neutral gas column density and distance. In Figure 4, we show the relations between $\mathrm{O}$ VII $\mathrm{K}_{\alpha} \mathrm{EW}$ and galactic latitude (left panel) and longitude (right panel). In the left panel, for $l$ in the range of 50 to 180 degree, the mean EW is less than 20 $\mathrm{m} \AA$, while toward the Galactic center for $l$ in the range of 0 to 30 degree, the mean $\mathrm{EW}$ is larger than $20 \mathrm{m \AA}$. For the right panel, a large part of the sample has coordinates toward the galactic center with coordinates $|b|$ in the range of 0 to 10 degree, however, the tendency for the EWs along with $b$ is rather weak. At higer latitude $|b|>20$, the EWs slightly increase with $|b|$, while at lower latitude, there is a large scatter of EWs.

In Figure 6 we show the measured $\mathrm{O}$ VII $\mathrm{K}_{\alpha}$ EW as a function of the Galactic hydrogen absorption $\mathrm{N}_{\mathrm{H}}$. It could be found the EWs likely show little variation with $\mathrm{N}_{\mathrm{H}}$ and there is no clear correlation between the EWs and $\mathrm{N}_{\mathrm{H}}$. Assuming a mean O VII EW of $20 \mathrm{m \AA}$ and a Doppler-b parameter of $100 \mathrm{~km} \mathrm{~s}^{-1}$, we could obtain an O VII column density of about $2 \times 10^{16} \mathrm{~cm}^{-2}$. Assuming solar abundance and all oxygen of the hot gas would be in the ionization stage of O VII, the total hydrogen column density of the hot gas would be $4.1 \times 10^{19} \mathrm{~cm}^{-2}$. By taking the ratio of the hot gas and neutral gas column density, we could find the relative fraction with neutral hydrogen would be in the range of 0.4 to 0.004 .

To investigate how the hot ISM distributes around the disk, we show the $\mathrm{O}$ VII $\mathrm{K}_{\alpha} \mathrm{EW}$ vs. the target distance in Figure 5. In general the EWs of the targets show a very weak and positive correlation, and also show scatters by a factor of a few or more among targets at similar distances. For targets with distance larger than $10 \mathrm{kpc}$, their EWs are mostly greater than $10 \mathrm{~mA}$, and with distance larger than $50 \mathrm{kpc}$, their EWs are greater than $20 \mathrm{m \AA}$. While for targets with distance less than $5 \mathrm{kpc}$, there are at least 2 objects with $\mathrm{EW}>30 \mathrm{~mA}$. However, due to large scattering, the trend between EW and distance is not obvious, and at small distance this large scatters coule be a result of the intrinsic absorption contamination.

We have calculated the dependance of the line EW on the galactic neutral absorption, target distance and the galactic latitude and longitude. Briefly, we first use software package ASURV Rev 1.2 (Isobe \& Feigelson 1990; Lavalley et al. 1992), which implements the methods presented in Isobe et al. (1986) and is a particular useful tool for correlation analysis of censored astronomical data, i.e., non-detections or detection limits. Then we characterize a correlation with a simple log-log linear relation, together with the dispersion around this relation from the root mean square (RMS) of the data. The corresponding RMS could give the probabilistic nature of the correlation and the measurement uncertainties. We listed the probability of correlation by chance for three correlation tests: Cox hazard model, generalized Kendall's tau, and Spearman's rho in Table 3 column 1, 2 , and 3, respectively. A correlation exists if the probability is less than $5 \%$. In general, the probability for all correlations are much larger than 5\%. In column 4 and 5 , we list the index of the fitted $\log$-log linear relation and the corresponding RMS, respectively. The index is quite close to zero, and the correlations are significantly sub-linear. With all these in consideration, we may then conclude that EW has no correlation with the galactic neutral absorption, the target distance or the galactic coordinates.

If the hot gas probed by the O VII absorption line is uniformly distributed in the ISM, one would expect the detected O VII column density may show some correlations with physical properties such as the the distance and/or coordinates of the background targets. Assuming the absorption line is in the linear part of the curve-ofgrowth, such correlations can be presented as the correlations between the line EW and these physical quantities. The non-detection of the correlations may suggest the line is in the non-linear part of the curve-of-growth and we do not have strong constraint on the line Doppler- 

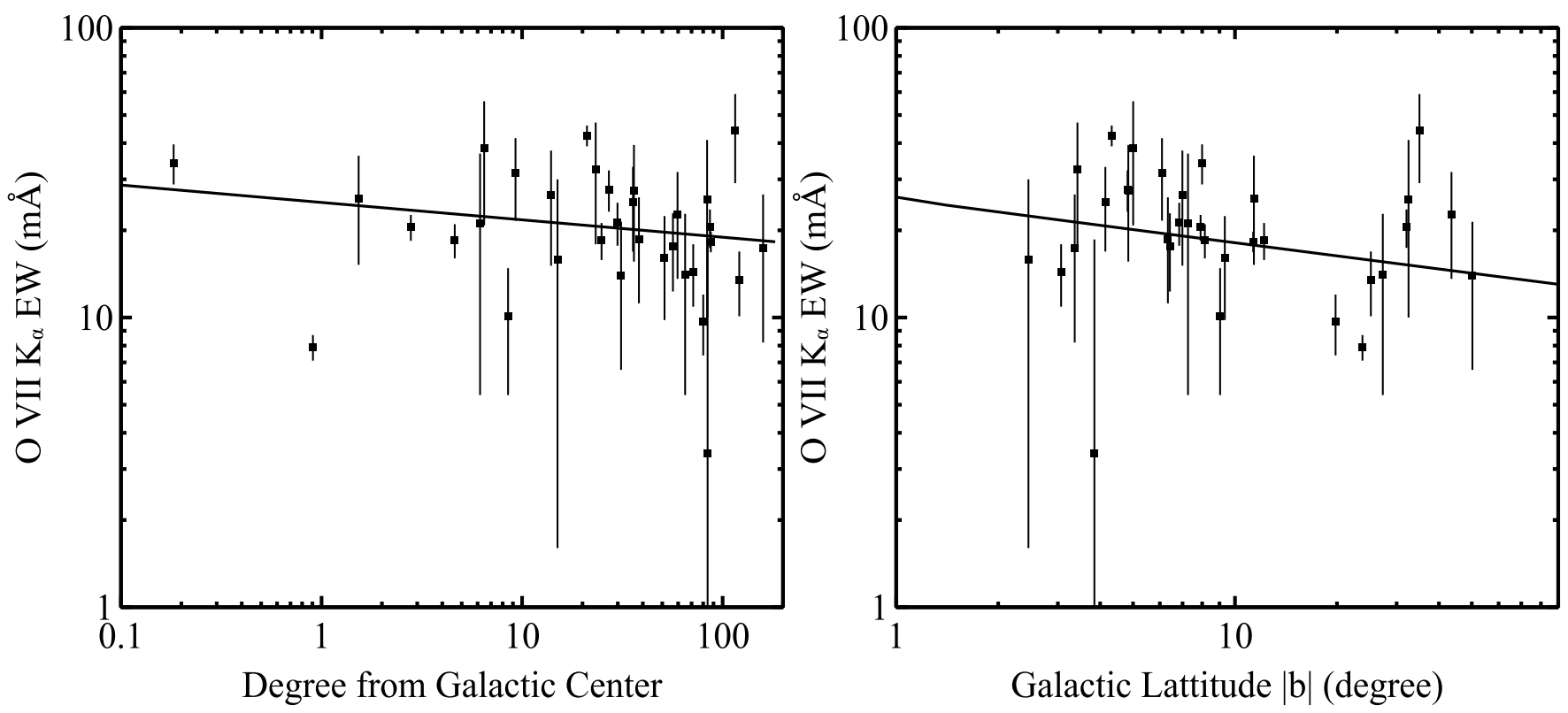

Figure 4. O VII $\mathrm{K}_{\alpha} \mathrm{EW}$ against the galactic longitude (left panel) and latitude (right panel).

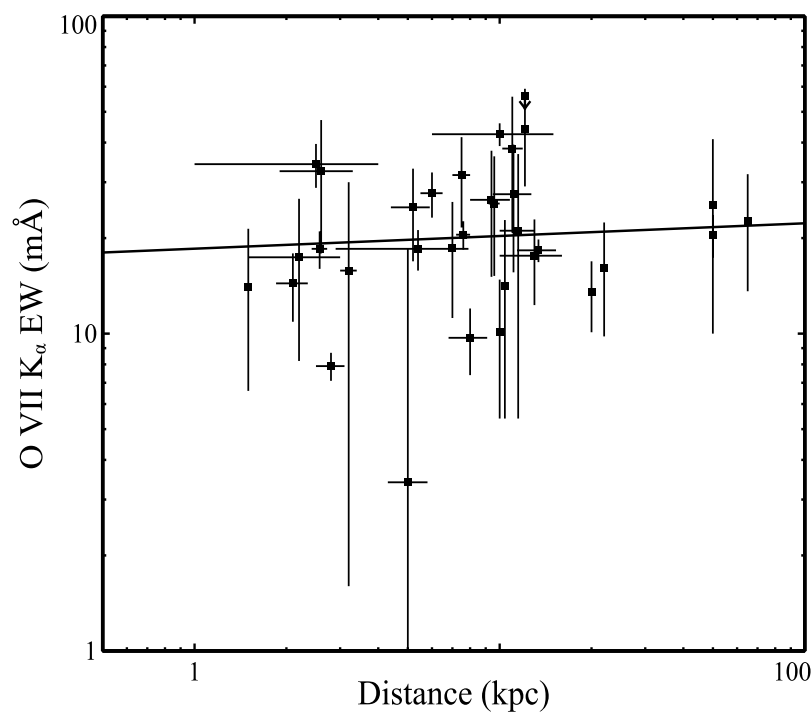

Figure 5. O VII $\mathrm{K}_{\alpha} \mathrm{EW}$ as function of distance.

b parameter. However, it is unlikely caused by the a non-uniform distribution of the hot gas properties, such as temperature, metallicity, or density, because of uniform distribution of the O VII absorption lines. Besides of these, the contamination of the circumstellar material, and the historical supernova activity (Ferriere 2001) could also introduce absorption to the lines.

One example is studying spatially closed targets for which the ISM distribution along the sightline towards these targets should be similar. We searched our sample for targets close to each other by their relative $l$ and $b$ within 5 degree. First we find that two targets SAX J1808.4-3658 and 4U 1820-30 show comparable O VII EW, whereas their distance differ by three times. The less distant target XTE J1817-330 has EW much larger than these two targets even though all three targets are spatial close to each other. Similarly in another group the EW for 4U 1636-54, with error bar taking into

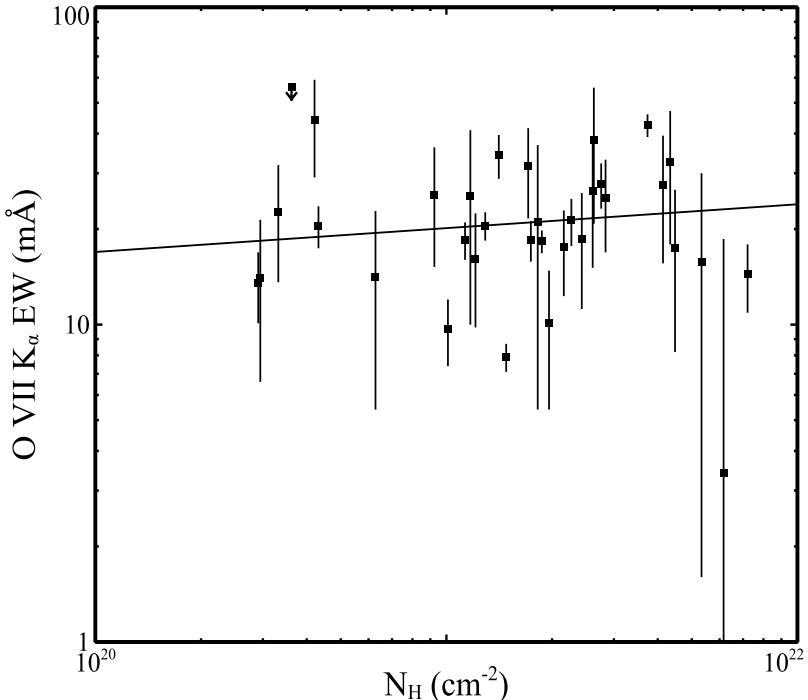

Figure 6. EWs of O VII $\mathrm{K}_{\alpha}$ line compared to galactic hydrogen absorption $\mathrm{N}_{\mathrm{H}}$. The $\mathrm{N}_{\mathrm{H}}$ is adopted from Kalberla et al. (2005)

consideration, is comparable with that of XTE J1650500, while $4 \mathrm{U}$ 1636-54 has a distance twice far away. Even though there are still too few pairs to draw definitive conclusions, this result may suggest that in some cases the intrinsic absorption dominates the ISM contribution.

\subsection{Comments on Some Individual Targets}

We briefly discuss several individual targets with newly detected O VII absorption lines at more than $3 \sigma$ significance level. In our sample, we find 16 targets showing line significance at more than $3 \sigma$ level, of which 4 targets are newly detected, Sco X-1, Swift J1753.5-0127, MAXI J0556-332 and MAXI J1910-057.

Sco $\mathrm{X}-1$ - This target is one of the brightest X-ray sources in the sky. With a high signal-to-noise ratio spectrum we can achieve much better constraints of the absorption line properties. Previous studies based on 
Table 3

Correlation Test

\begin{tabular}{llcccc}
\hline \hline Correlation & Cox Hazard & Kendall Tau & Spearman Rho & $\Gamma$ & RMS (dex) \\
\hline EW vs. $N_{\mathrm{H}}$ & $40 \%$ & $87 \%$ & $87 \%$ & $0.075 \pm 0.001$ & $0.23 \pm 0.06$ \\
\hline EW vs. Distance & $20 \%$ & $21 \%$ & $19 \%$ & $0.04 \pm 0.12$ & $0.26 \pm 0.07$ \\
\hline EW vs. $l$ & $36 \%$ & $32 \%$ & $34 \%$ & $-0.06 \pm 0.08$ & $0.26 \pm 0.07$ \\
\hline EW vs. $|b|$ & $53 \%$ & $40 \%$ & $37 \%$ & $-0.15 \pm 0.18$ & $0.26 \pm 0.08$ \\
\hline
\end{tabular}

absorption lines mostly focus on the low ionization absorption lines or absorption edges, and our measurement is the first report on the O VII detection (García et al. 2011; de Vries \& Costantini 2009). The O VII line in Sco X-1 is detected at $9.9 \sigma$ significance level and the line shift velocity is very low (less than $100 \mathrm{~km} \mathrm{~s}^{-1}$ ).

Swift J1753.5-0127 - This is also a LMXB discovered with the Swift/BAT on May,2005 (Palmer et al. 2005). Mostafa et al. (2013) report the discovery of broad emission lines of N VII and O VIII in the RGS spectrum. They attributed this feature as a result of reflection of $\mathrm{X}$-ray photons off accretion disk.

MAXI J0556-332 - This is a X-ray transient. Maitra et al. (2011) reported the detection of a strong emission line near $24.8 \AA$, identified as N VII. So far there is no report of absorption lines produced by an outflow. We detect the O VII absorption with $4 \sigma$ significance level.

MAXI J1910-057 - The source was first detected by the Swift/BAT (Krimm et al. 2012) on May, 2012 and simultaneously by the MAXI telescope (Usui et al. 2012). The observed spectra are typical of an LMXB Charles et al. (2012) indicating that it is comparable with other neutron star/black hole soft X-ray transients, but the distance is still unclear yet. The O VII line in this target is first reported here with a blueshift velocity of $205 \mathrm{~km} \mathrm{~s}^{-1}$, an EW of $21.3 \mathrm{m \AA}$ and a significance of 6.0 .

$4 \mathrm{U}$ 0513-40 - This is an ultracompact X-ray binary in the globular cluster NGC 1851. This target was only observed by XMM-Newton once in April, 2003. Previous X-ray observations did not report any detection of O VII absorption line and in our work we found this line having shift-velocity of $-595 \mathrm{~km} \mathrm{~s}^{-1}$ and detection significance at $2.9 \sigma$ level. Bt its high shift velocity, this absorption line could be a good indicator of an circumstellar medium origin.

\section{SUMMARY}

Understanding the properties of the hot ionized medium has profound impact on the study stellar feedback and the multiphase structure of the ISM. In this work, we perform a survey in the XMM-Newton archive for searching O VII absorption lines in the spectra of Galactic X-ray sources. We summarize our findings here.

- We analyzed 33 Galactic XRBS with 29 targets are LMXBs and 4 targets are HMXBs. Most targets are located at a distance if within $20 \mathrm{kpc}$, in the Galactic disk. 16 targets have O VII detection at more than $3 \sigma$, among which 4 are newly discovered in this work. We find one targets showing shiftvelocity larger than $500 \mathrm{~km} \mathrm{~s}^{-1}$ indicating of an circumsteller medium origin.

- We fitted the $\mathrm{O}$ VII $\mathrm{K}_{\alpha}$ transitions with a Voigtprofile based line model. We find that most $\mathrm{K}_{\alpha}$ lines have an EW of $20 \mathrm{~m} \AA$, with a range of $10-40$ $\mathrm{m} \AA$.

- We find that the detection fractions, or the sky covering fractions, increase steadily as the increasing CPRE. For targets with CPRE more than about 400 counts the $3 \sigma$ detection fraction reaches $100 \%$ regardless of the direction of the XRBs. Such detection rate implies a uniform distribution of the hot gas in all directions.

- The EWs of the O VII line do not correlate with the galactic neutral absorption $\mathrm{N}_{\mathrm{H}}$. The fraction of the content of hot medium to that of neutral phase is estimated in the range of 0.004 to 0.4 . We do not find any strong correlations between O VII EW and target distance, so as to the targets coordinates. The EW for targets at similar distance show large scatters and the difference could be as large as one order of magnitude. The reasons for the non-correlation could be the contribution of circumstellar material or the uncertainties on the column density measurements, as we do not have constraints on the Doppler-b parameter, so EWs may not exactly reflect the column density distribution.

The highly ionized, $\mathrm{z}=0 \mathrm{O}$ VII absorption lines detected in the X-ray spectra of background AGNs, along with the detections in Galactic XRBs could help to constrain the distribution of hot ISM with a broad range of temperature around $10^{6} \mathrm{~K}$. We briefly compare our results with the AGN O VII line survey by Fang et al. (2015), and we find there are some similarities of the line properties. The detection fractions, in both surveys, increase steadily as the increasing CPRE, which suggesting a uniform distribution of the O VII absorbers. The histogram of $\mathrm{K}_{\alpha}$ lines in both cases have an median EW of $20 \mathrm{~m} \AA$, and there are also no correlations between the EWs and the targets coordinates.

By considering the different coordinates and distances or covering area and absorbing depth of the AGNs and XRBs, we could find that the XRBs have a large fraction of contaminations intrinsically contributing to the O VII absorption. But how much the XRBs locally contribute to the absorption still needs more investigations. Further theoretical modeling in combine with X-ray emission study, and X-ray absorption study will be presented in a later paper.

We thank Drs. David Buote and Daniel Wang for helpful discussion, This work is supported by National Key R\&D Program of China No. 2017YFA0402600, NSFC grants No. 11525312, 11333004, 11443009, and U1531130. TF is also supported by the Specialized Re- 
search Fund for the Doctoral Program of Higher Education (SRFDP; No. 20130121110009).

\section{REFERENCES}

Anderson, M. E., \& Bregman, J. N. 2011, ApJ, 737, 22

Anderson, M. E., Churazov, E., \& Bregman, J. N. 2016, MNRAS, 455, 227

Arnaud, K. A. 1996, in Astronomical Society of the Pacific Conference Series, Vol. 101, Astronomical Data Analysis Software and Systems V, ed. G. H. Jacoby \& J. Barnes, 17

Bogdan, A., Forman, W. R., Kraft, R. P., \& Jones, C. 2013, arXiv preprint arXiv:1306.0643

Bogdan, A., Forman, W. R., Vogelsberger, M., et al. 2012, arXiv preprint arXiv:1212.0541

Bregman, J. N., \& Lloyd-Davies, E. J. 2007, ApJ, 669, 990

Buote, D. A., Zappacosta, L., Fang, T., et al. 2009, ApJ, 695, 1351

Cabot, S. H. C., Wang, Q. D., \& Yao, Y. 2013, MNRAS, 431, 511

Cackett, E. M., Miller, J. M., Raymond, J., et al. 2008, ApJ, 677, 1233

Cappelluti, N., Ranalli, P., Roncarelli, M., et al. 2012, MNRAS, 427,651

Caraveo, P. A., De Luca, A., Mignani, R. P., \& Bignami, G. F. 2001, ApJ, 561, 930

Charles, P., Cornelisse, R., \& Casares, J. 2012, The Astronomer's Telegram, 4210

Collins, J. A., Shull, J. M., \& Giroux, M. L. 2005, ApJ, 623, 196

Costantini, E., Pinto, C., Kaastra, J. S., et al. 2012, A\&A, 539, A32

Cottam, J., Paerels, F., \& Mendez, M. 2002, Nature, 420, 51

Crain, R. A., McCarthy, I. G., Frenk, C. S., Theuns, T., \& Schaye, J. 2010, MNRAS, 407, 1403

Dai, X., Anderson, M. E., Bregman, J. N., \& Miller, J. M. 2012, The Astrophysical Journal, 755, 107

de Vries, C. P., \& Costantini, E. 2009, A\&A, 497, 393

Fang, T., Bullock, J., \& Boylan-Kolchin, M. 2013, ApJ, 762, 20

Fang, T., Buote, D. A., Bullock, J. S., \& Ma, R. 2015, ArXiv e-prints

Fang, T., Buote, D. A., Humphrey, P. J., et al. 2010, ApJ, 714, 1715

Fang, T., Mckee, C. F., Canizares, C. R., \& Wolfire, M. 2006, ApJ, 644, 174

Fang, T., Sembach, K. R., \& Canizares, C. R. 2003, ApJ, 586, L49

Feldmann, R., Hooper, D., \& Gnedin, N. Y. 2013, ApJ, 763, 21

Ferriere, K. M. 2001, Reviews of Modern Physics, 73, 1031

Fukugita, M., \& Peebles, P. J. E. 2006, ApJ, 639, 590

Futamoto, K., Mitsuda, K., Takei, Y., Fujimoto, R., \& Yamasaki, N. Y. 2004, ApJ, 605, 793

Galeazzi, M., Chiao, M., Collier, M. R., et al. 2014, Nature, 512, 171

Galloway, D. K., Psaltis, D., Muno, M. P., \& Chakrabarty, D. 2006, ApJ, 639, 1033

García, J., Ramírez, J. M., Kallman, T. R., et al. 2011, ApJ, 731, L15

Gatto, A., Fraternali, F., Read, J. I., et al. 2013, MNRAS, 433, 2749

Gatuzz, E., García, J., Mendoza, C., et al. 2013, ApJ, 768, 60

Grcevich, J., \& Putman, M. E. 2009, ApJ, 696, 385

Hagihara, T., Yao, Y., Yamasaki, N. Y., et al. 2010, PASJ, 62, 723

Helton, L. A., Woodward, C. E., Vanlandingham, K., \& Schwarz, G. J. 2008, Central Bureau Electronic Telegrams, 1379, 1

Henley, D. B., \& Shelton, R. L. 2013, ApJ, 773, 92

Hessels, J. W. T., Ransom, S. M., Stairs, I. H., Kaspi, V. M., \& Freire, P. C. C. 2007, ApJ, 670, 363

Hodges-Kluck, E. J., \& Bregman, J. N. 2013, ApJ, 762, 12

Hynes, R. I., Steeghs, D., Casares, J., Charles, P. A., \& O'Brien, K. 2004, ApJ, 609, 317

in't Zand, J. J. M., Kuulkers, E., Verbunt, F., Heise, J., \& Cornelisse, R. 2003, A\&A, 411, L487

in't Zand, J. J. M., Cornelisse, R., Kuulkers, E., et al. 2001, A\&A, 372, 916

Isobe, T., \& Feigelson, E. D. 1990, in Bulletin of the American Astronomical Society, Vol. 22, Bulletin of the American Astronomical Society, 917-918

Isobe, T., Feigelson, E. D., \& Nelson, P. I. 1986, ApJ, 306, 490
Jonker, P. G., \& Nelemans, G. 2004, MNRAS, 354, 355

Kalberla, P. M. W., Burton, W. B., Hartmann, D., et al. 2005 A\&A, 440, 775

Kaplan, D. L., van Kerkwijk, M. H., \& Anderson, J. 2007, ApJ, 660,1428

Kong, A. K. H., Homer, L., Kuulkers, E., Charles, P. A., \& Smale, A. P. 2000, MNRAS, 311, 405

Krimm, H. A., Barthelmy, S. D., Baumgartner, W., et al. 2012, The Astronomer's Telegram, 4139

Kuulkers, E., den Hartog, P. R., in't Zand, J. J. M., et al. 2003, A\&A, 399, 663

Lallement, R. 2004, A\&A, 422, 391

Lavalley, M., Isobe, T., \& Feigelson, E. 1992, in Astronomical Society of the Pacific Conference Series, Vol. 25, Astronomical Data Analysis Software and Systems I, ed. D. M. Worrall, C. Biemesderfer, \& J. Barnes, 245

Lehner, N., Howk, J. C., Thom, C., et al. 2012, MNRAS, 424, 2896

Li, J.-T., \& Wang, Q. D. 2013, Monthly Notices of the Royal Astronomical Society, 428, 2085

Liao, J.-Y., Zhang, S.-N., \& Yao, Y. 2013, ApJ, 774, 116

Luo, Y., \& Fang, T. 2014, ApJ, 780, 170

Maitra, D., Miller, J. M., Raymond, J. C., \& Reynolds, M. T. 2011, ApJ, 743, L11

Maller, A. H., \& Bullock, J. S. 2004, MNRAS, 355, 694

Marinacci, F., Fraternali, F., Nipoti, C., et al. 2011, MNRAS, 415,1534

Mason, K. O., \& Cordova, F. A. 1982, ApJ, 262, 253

Miller, J. M., Raymond, J., Fabian, A. C., et al. 2004, ApJ, 601, 450

Miller, M. J., \& Bregman, J. N. 2013, ApJ, 770, 118

Mo, H. J., \& Mao, S. 2002, MNRAS, 333, 768

Mostafa, R., Mendez, M., Hiemstra, B., et al. 2013, MNRAS, 431, 2341

Muno, M. P., Chakrabarty, D., Galloway, D. K., \& Savov, P. 2001, ApJ, 553, L157

Nicastro, F., Zezas, A., Drake, J., et al. 2002, ApJ, 573, 157

Nowak, M. A., Juett, A., Homan, J., et al. 2008, ApJ, 689, 1199

Ntormousi, E., \& Sommer-Larsen, J. 2010, MNRAS, 409, 1049

Paerels, F., Brinkman, A. C., van der Meer, R. L. J., et al. 2001 ApJ, 546, 338

Palmer, D. M., Barthelmey, S. D., Cummings, J. R., et al. 2005, The Astronomer's Telegram, 546, 1

Petrucci, P.-O., Cabanac, C., Corbel, S., Koerding, E., \& Fender, R. 2014, A\&A, 564, A37

Pietrzyński, G., Graczyk, D., Gieren, W., et al. 2013, Nature, 495, 76

Pinto, C., Costantini, E., Fabian, A. C., Kaastra, J. S., \& in't Zand, J. J. M. 2014, A\&A, 563, A115

Pinto, C., Kaastra, J. S., Costantini, E., \& de Vries, C. 2013 A\&A, 551, A25

Putman, M., Peek, J., \& Joung, M. 2012, arXiv preprint arXiv: 1207.4837

Rasmussen, A., Kahn, S. M., \& Paerels, F. 2003, in Astrophysics and Space Science Library, Vol. 281, The IGM/Galaxy Connection. The Distribution of Baryons at $\mathrm{z}=0$, ed. J. L. Rosenberg \& M. E. Putman, 109

Rau, A., Greiner, J., \& Filgas, R. 2011, The Astronomer's Telegram, 3140, 1

Sakai, K., Yao, Y., Mitsuda, K., et al. 2014, PASJ, 66, 83

Sala, G., \& Greiner, J. 2006, The Astronomer's Telegram, 791, 1

Sala, G., Greiner, J., Ajello, M., Bottacini, E., \& Haberl, F. 2007, A\&A, 473, 561

Savolainen, P., Hannikainen, D. C., Vilhu, O., et al. 2009 MNRAS, 393, 569

Schulz, N. S., Cui, W., Canizares, C. R., et al. 2002, ApJ, 565, 1141

Sembach, K. R., Wakker, B. P., Savage, B. D., et al. 2003, ApJS, 146,165

Sokołowska, A., Mayer, L., Babul, A., Madau, P., \& Shen, S. 2016, ApJ, 819, 21

Spitzer, Jr., L. 1956, ApJ, 124, 20

Stinson, G. S., Brook, C., Prochaska, J. X., et al. 2012, MNRAS, 425,1270

Tetzlaff, N., Eisenbeiss, T., Neuhäuser, R., \& Hohle, M. M. 2011, MNRAS, 417, 617

Trimble, V. 1973, PASP, 85, 579 
Tüllmann, R., Pietsch, W., Rossa, J., Breitschwerdt, D., \& Dettmar, R.-J. 2006, A\&A, 448, 43

Usui, R., Nakahira, S., Tomida, H., et al. 2012, The Astronomer's Telegram, 4140

van Peet, J. C. A., Costantini, E., Méndez, M., Paerels, F. B. S., \& Cottam, J. 2009, A\&A, 497, 805

Verner, D. A., Verner, E. M., \& Ferland, G. J. 1996, Atomic Data and Nuclear Data Tables, 64, 1

Wang, Q. D., Yao, Y., Tripp, T. M., et al. 2005, ApJ, 635, 386

Wang, Z., \& Chakrabarty, D. 2004, ApJ, 616, L139

White, S. D. M., \& Frenk, C. S. 1991, ApJ, 379, 52

Yao, Y., Nowak, M. A., Wang, Q. D., Schulz, N. S., \& Canizares, C. R. 2008, The Astrophysical Journal Letters, 672, L21

Yao, Y., Schulz, N. S., Gu, M. F., Nowak, M. A., \& Canizares, C. R. 2009, ApJ, 696, 1418
Yao, Y., Wang, Q., Hagihara, T., et al. 2009, The Astrophysical Journal, 690, 143

Yao, Y., \& Wang, Q. D. 2005, The Astrophysical Journal, 624, 751

Yao, Y., \& Wang, Q. D. 2006, ApJ, 641, 930

-. 2007, ApJ, 658, 1088

Yao, Y., Wang, Q. D., Hagihara, T., et al. 2009, ApJ, 690, 143

Ziółkowski, J. 2005, MNRAS, 358, 851

Zurita, C., Durant, M., Torres, M. A. P., et al. 2008, ApJ, 681, 1458 
Table 4

The X-ray Targets Sample

\begin{tabular}{|c|c|c|c|c|c|c|c|c|c|c|c|c|c|}
\hline Source & Type & $\begin{array}{l}\mathrm{N}_{\mathrm{H}} \\
\left(\mathrm{cm}^{-2}\right)\end{array}$ & 1 & $\mathrm{~b}$ & $\begin{array}{l}\text { Distance } \\
(\mathrm{kpc})\end{array}$ & $\begin{array}{l}\text { Exp. } \\
\text { (ksec) }\end{array}$ & Counts & $\begin{array}{l}\log \left(\mathrm{N}_{\text {OVII }}\right) \\
\left(\mathrm{cm}^{-2}\right)\end{array}$ & $\begin{array}{l}\text { Velocity } \\
\left(\mathrm{km} \mathrm{s}^{-1}\right)\end{array}$ & $\begin{array}{l}\text { Doppler-b } \\
\left(\mathrm{km} \mathrm{s}^{-1}\right)\end{array}$ & $\begin{array}{l}\mathrm{EW}\left(\mathrm{K}_{\alpha}\right) \\
(\mathrm{mA})\end{array}$ & $\begin{array}{l}\mathrm{S} / \mathrm{N} \\
(\sigma)\end{array}$ & $C /$ dof \\
\hline Sco X-1 & LMXB & $1.48 \mathrm{E}+21$ & 359.094 & 23.784 & $2.8_{-0.3}^{+0.3}[6]$ & 36.8 & 16612.7 & $15.8_{-0.4}^{+1.2}$ & $15.4_{-93.4}^{+98.9}$ & $42.8_{-22.8}^{+274.2}$ & $7.9 \pm 0.8$ & 9.9 & $972 / 662$ \\
\hline Cygnus $\mathrm{X}-2$ & LMXB & $1.87 \mathrm{E}+21$ & 87.328 & -11.316 & $13.4_{-2}^{+1.9}[6]$ & 199.9 & 3089.7 & $16.3_{-0.5}^{-0.4}$ & $145.5_{-108.3}^{+108.4}$ & $78.9_{-50.9}^{+221.8}$ & $18.3 \pm 1.5$ & 12.2 & $1190 / 971$ \\
\hline XTE J1817-330 & LMXB & $1.41 \mathrm{E}+21$ & 359.817 & -7.996 & $2.5_{-1.5}^{+1.5}[6]$ & 18.0 & 1379.4 & $16.2_{-0.2}^{+0.5}+0.2$ & $2.3_{-304.2}^{+271.3}$ & $300.0_{-300.0}^{+50.9}$ & $34.2 \pm 5.4$ & 6.3 & $219 / 206$ \\
\hline $4 \mathrm{U} 1820-30$ & LMXB & $1.29 \mathrm{E}+21$ & 2.788 & -7.914 & $7.6_{-0.4}^{+0.4}[24]$ & 76.4 & 1185.2 & $16.8_{-0.9}^{+0.9}$ & $56.4_{-110.3}^{+108.0}$ & $75.4_{-35.2}^{+224.6}$ & $20.5 \pm 2.1$ & 9.8 & $347 / 327$ \\
\hline GX $339-4$ & LMXB & $3.74 \mathrm{E}+21$ & 338.939 & -4.326 & $10_{-4}^{+5}[1]$ & 443.1 & 1017.9 & $16.7_{-0.1}^{+0.1}$ & $\begin{array}{l}-71.3_{-80.3}^{+80.5} \\
-80\end{array}$ & $202.2_{-43.3}^{+60.7}$ & $42.5 \pm 3.5$ & 12.1 & $1384 / 1354$ \\
\hline SAX J1808.4-3658 & LMXB & $1.13 \mathrm{E}+21$ & 355.385 & -8.148 & $2.57_{-0.15}^{+0.15}[2]$ & 58.8 & 910.6 & $15.9_{-0.1}^{+0.6}$ & $\begin{array}{r}-80.3 \\
-118.1_{-216.5}^{+186.5}\end{array}$ & $300.0_{-280.0}^{+0.0}$ & $18.5 \pm 2.5$ & 7.4 & $289 / 206$ \\
\hline Swift J1753.5-0127 & LMXB & $1.74 \mathrm{E}+21$ & 24.898 & 12.186 & $5.4_{-2.5}^{+2.5}[11]$ & 118.1 & 798.8 & $15.9_{-0.2}^{+0.1}$ & $\begin{array}{r}-185.4_{-25.9}^{+220.3} \\
-25.9\end{array}$ & $300.0_{-280.0}^{+0.0}$ & $18.5 \pm 2.7$ & 6.9 & $572 / 520$ \\
\hline EXO 0748-676 & LMXB & $1.01 \mathrm{E}+21$ & 279.975 & -19.811 & $8{ }_{-1.2}^{+1.1}[15]$ & 382.9 & 691.8 & $16.0_{-0}^{+1:}$ & $24.5_{-170.5}^{+20.2 .9}$ & $46.2_{-26.2}^{+253.8}$ & $9.7 \pm 2.3$ & 4.2 & $1877 / 1433$ \\
\hline Cygnus X-1 & HMXB & $7.21 \mathrm{E}+21$ & 71.335 & 3.067 & $2.1{ }_{-0.25}^{+0.25}[16]$ & 194.4 & 595.0 & $15.8_{-0.3}^{+1.7}$ & $-494.5_{-5.5}^{+379.6}$ & $251.4_{-231.4}^{+48.6}$ & $14.4 \pm 3.5$ & 4.1 & $1629 / 1472$ \\
\hline MAXI J0556-332 & LMXB & $2.91 \mathrm{E}+20$ & 238.940 & -25.183 & $20[5]$ & 71.7 & 576.4 & $16.1_{-0.7}^{+1.3}$ & $84.6_{-274}^{+259}$ & $68.2_{-48.2}^{+231.8}$ & $13.5 \pm 3.4$ & 4.0 & $322 / 369$ \\
\hline LMC X-3 & HMXB & $4.32 \mathrm{E}+20$ & 273.576 & -32.082 & $49.97 \quad[17]$ & 146.1 & 564.0 & $16.5_{-0.7}^{+1.2}$ & $92.9_{-137.2}^{+139.5}$ & $85.6_{-46.5}^{+214.4}$ & $20.5 \pm 3.1$ & 6.6 & $960 / 955$ \\
\hline MAXI J1910-057 & LMXB & $2.27 \mathrm{E}+21$ & 29.903 & -6.844 & - & 51.2 & 529.1 & $15.9_{-0}^{+0 .}$ & $\begin{array}{r}-205.4+25.5 \\
-250.2\end{array}$ & $300.0_{-271.4}^{-46.5}$ & $21.3 \pm 3.6$ & 5.9 & $225 / 120$ \\
\hline $4 \mathrm{U} 1636-54$ & LMXB & $2.76 \mathrm{E}+21$ & 332.915 & -4.818 & $6{ }_{-0.5}^{+0.5}[9]$ & 245.1 & 390.9 & $16.7_{-0}^{+1 .}$ & $\begin{array}{r}-170.8_{-164.4}^{+1750.2} \\
\end{array}$ & $113.0_{-59.2}^{+271.0}$ & $27.7 \pm 4.5$ & 6.2 & $1064 / 1067$ \\
\hline $4 \mathrm{U} 1728-16$ & LMXB & $1.96 \mathrm{E}+21$ & 8.513 & 9.038 & $10[10]$ & 32.1 & 234.0 & $15.9_{-0.9}^{+1.6}$ & $\begin{array}{r}-364.7_{-135.3}^{+417.8} \\
\end{array}$ & $55.1_{-35.1}^{+249.2}$ & $10.1 \pm 4.7$ & 2.1 & $452 / 401$ \\
\hline $4 \mathrm{U} 1254-690$ & LMXB & $2.16 \mathrm{E}+21$ & 303.482 & -6.424 & $13_{-3}^{+3}[13]$ & 214.0 & 215.9 & $15.9_{-0.4}^{+1.8}$ & $\begin{array}{l}-35.3_{-340.4}^{+416.9} \\
-14.3\end{array}$ & $203.5_{-183.5}^{+96.5}$ & $17.6 \pm 5.3$ & 3.3 & $685 / 664$ \\
\hline Aql X-1 & LMXB & $2.84 \mathrm{E}+21$ & 35.718 & -4.143 & $5.2_{-0.8}^{+0.7}[6]$ & 95.1 & 149.1 & $16.0_{-0.3}^{+0.4}$ & $248.4{ }_{-520.0}^{+340.4}$ & $299.4_{-279.4}^{+0.6}$ & $25.0 \pm 8.1$ & 3.1 & $405 / 386$ \\
\hline $4 \mathrm{U} 1957+11$ & LMXB & $1.21 \mathrm{E}+21$ & 51.309 & -9.331 & $22[21]$ & 44.8 & 132.4 & $16.4_{-1 .}^{+1 .}$ & $\begin{array}{r}-520.0 \\
-281.5_{-218.5}^{+584.1}\end{array}$ & $69.5_{-49.5}^{+230.5}$ & $16.1 \pm 6.3$ & 2.6 & $164 / 162$ \\
\hline $4 \mathrm{U} 1543-62$ & LMXB & $2.43 \mathrm{E}+21$ & 321.755 & -6.337 & $7[3]$ & 49.1 & 113.2 & $16.5_{-1}^{+1 .}$ & $\begin{array}{r}-218.5 \\
-189.3_{-310.7}^{+307.9}\end{array}$ & $71.3_{-51.3}^{-49.5}$ & $18.6 \pm 7.4$ & 2.5 & $190 / 207$ \\
\hline Swift J1357.2-0933 & LMXB & $2.95 \mathrm{E}+20$ & 328.839 & 50.210 & $1.5[19]$ & 27.8 & 108.5 & $16.0_{-1.0}^{+1.7}$ & $48.5_{-548.5}^{+451.5}$ & $90.8_{-70.8}^{+209.2}$ & $14.0 \pm 7.4$ & 1.9 & $153 / 163$ \\
\hline GS $1826-238$ & LMXB & $1.71 \mathrm{E}+21$ & 9.272 & -6.088 & $7.5_{-0.5}^{+0.5}[6]$ & 228.2 & 104.7 & $16.2_{-0.4}^{+0.0}$ & $\begin{array}{l}-437.4_{-64.6}^{+58.5}+52.8 \\
-50\end{array}$ & $300.0_{-280.0}^{+0.8}$ & $31.6 \pm 10.0$ & 3.2 & $290 / 309$ \\
\hline $4 \mathrm{U} 2129+12$ & LMXB & $6.28 \mathrm{E}+20$ & 65.013 & -27.312 & $10.4[7]$ & 86.4 & 98.1 & $16.4_{-1.4}^{+1.0}+4$ & $\begin{array}{r}-133.4_{-366.6}^{+62.6} \\
+333.4\end{array}$ & $42.4_{-22.4}^{+257.6}$ & $14.1 \pm 8.7$ & 1.6 & $297 / 242$ \\
\hline $4 \mathrm{U} 1735-44$ & LMXB & $2.61 \mathrm{E}+21$ & 346.054 & -6.994 & $9.4_{-1.4}^{+1.4}[14]$ & 20.1 & 82.0 & $16.1_{-0.4}^{+0.3}$ & $-75.0_{-345.8}^{+475.0}$ & $300.0_{-193.7}^{+0.4}$ & $26.4 \pm 11.3$ & 2.3 & $170 / 163$ \\
\hline $4 \mathrm{U} 0614+09$ & LMXB & $4.48 \mathrm{E}+21$ & 200.877 & -3.364 & $2.2_{-0.7}^{+0.8}[20]$ & 23.4 & 74.0 & $16.5_{-1.5}^{+0.2}$ & $206.8_{-572.2}^{+293.8}$ & $72.3_{-52.3}^{+227.7}$ & $17.4 \pm 9.2$ & 1.9 & $232 / 229$ \\
\hline SMC X-1 & HMXB & $3.32 \mathrm{E}+20$ & 300.415 & -43.559 & $65 \quad[23]$ & 36.5 & 73.2 & $16.5_{-0.8}^{+1.2}$ & $318.8_{-571.4}^{+181.2}$ & $101.6_{-81.6}^{+198.4}$ & $22.7 \pm 9.1$ & 2.5 & $209 / 199$ \\
\hline GRO J1655-40 & LMXB & $5.33 \mathrm{E}+21$ & 344.982 & 2.456 & $3.2_{-0.2}^{+0.2}[6]$ & 113.8 & 70.7 & $15.8_{-0}^{+1}$ & $\begin{array}{r}-5117.2 \\
-230.5+269.5\end{array}$ & $61.1_{-41.1}^{+238.9}$ & $15.8 \pm 14.2$ & 1.1 & $351 / 331$ \\
\hline XTE J1650-500 & LMXB & $4.34 \mathrm{E}+21$ & 336.718 & -3.427 & $2.6_{-0.7}^{+0.7}[17]$ & 23.9 & 63.2 & $16.1_{-1.0}^{+1.6}$ & $228.1_{-435.1}^{+272.5}$ & $300.0_{-280.0}^{+0.1}$ & $32.5 \pm 14.6$ & 2.2 & $189 / 198$ \\
\hline Ser X-1 & LMXB & $4.14 \mathrm{E}+21$ & 36.118 & 4.842 & $11.1_{-1.6}^{+1.6}[4]$ & 64.2 & 57.4 & $17.1_{-1.5}^{+0.6}$ & $159.4_{-492.4}^{+340.6}$ & $95.8_{-75.8}^{+204.2}$ & $27.5 \pm 11.9$ & 2.3 & $253 / 278$ \\
\hline XB $1832-330$ & LMXB & $9.24 \mathrm{E}+20$ & 1.531 & -11.371 & $9.6_{-0.4}^{+0.4}[8]$ & 63.6 & 54.0 & $16.7_{-1.1}^{+1.0}$ & $\begin{array}{r}-412.6_{-287.5}^{+459.9} \\
-4.9\end{array}$ & $102.1_{-82.1}^{+198.0}$ & $25.7 \pm 10.5$ & 2.5 & $151 / 181$ \\
\hline $4 \mathrm{U} 0513-40$ & LMXB & $4.21 \mathrm{E}+20$ & 244.510 & -35.036 & $12.1_{-0.3}^{+0.3}[6]$ & 23.7 & 28.7 & $\ldots$ & $\ldots$ & $\ldots$ & $<56.1$ & $\ldots$ & $\ldots$ \\
\hline $4 \mathrm{U} 0513-40^{*}$ & $\ldots$ & $\ldots$ & $\ldots$ & $\ldots$ & $\ldots$ & $\ldots$ & $\ldots$ & $16.5_{-0.5}^{+0.4}$ & $-595 .{ }_{-5.0}^{+340.1}$ & $300.0_{-280.0}^{+0.0}$ & $44.1 \pm 15.0$ & 2.9 & $90 / 92$ \\
\hline LMC X-4 & HMXB & $1.17 \mathrm{E}+21$ & 276.335 & -32.529 & $49.97 \quad[12]$ & 43.7 & 28.7 & $16.8_{-1.8}^{+0.6}$ & $0.2_{-391.9}^{+383.50}$ & $97.1_{-77.9}^{+202.9}$ & $25.5 \pm 15.5$ & 1.6 & $100 / 92$ \\
\hline 4U $0919-54$ & LMXB & $6.16 \mathrm{E}+21$ & 275.852 & -3.845 & $5_{-0.7}^{+0.8}[18]$ & 39.7 & 26.3 & $15.8_{-0.8}^{+1.6}$ & $0.0+500.0$ & $97.5_{-77.5}^{+202.5}$ & $3.4 \pm 15.2$ & 0.2 & $73 / 103$ \\
\hline MXB $1659-29$ & LMXB & $1.82 \mathrm{E}+21$ & 353.827 & 7.266 & $11.5_{-1.5}^{+1.5}[6]$ & 30.6 & 24.8 & $16.4_{-1.4}^{+1.8}$ & $237.0_{-537}^{+263.0}$ & $95.1_{-75.1}^{+204.9}$ & $21.1 \pm 15.7$ & 1.3 & $83 / 82$ \\
\hline $4 \mathrm{U} 1746-371$ & LMXB & $2.63 \mathrm{E}+21$ & 353.531 & -5.005 & $11_{-0.8}^{+0.9}[22]$ & 71.3 & 22.9 & $17.1_{-1.3}^{+0.4}$ & $\begin{array}{r}-272.7{ }_{-227.3}^{+301.8} \\
\end{array}$ & $141.0_{-120.9}^{+159.0}$ & $38.3 \pm 17.5$ & 2.2 & $93 / 94$ \\
\hline
\end{tabular}



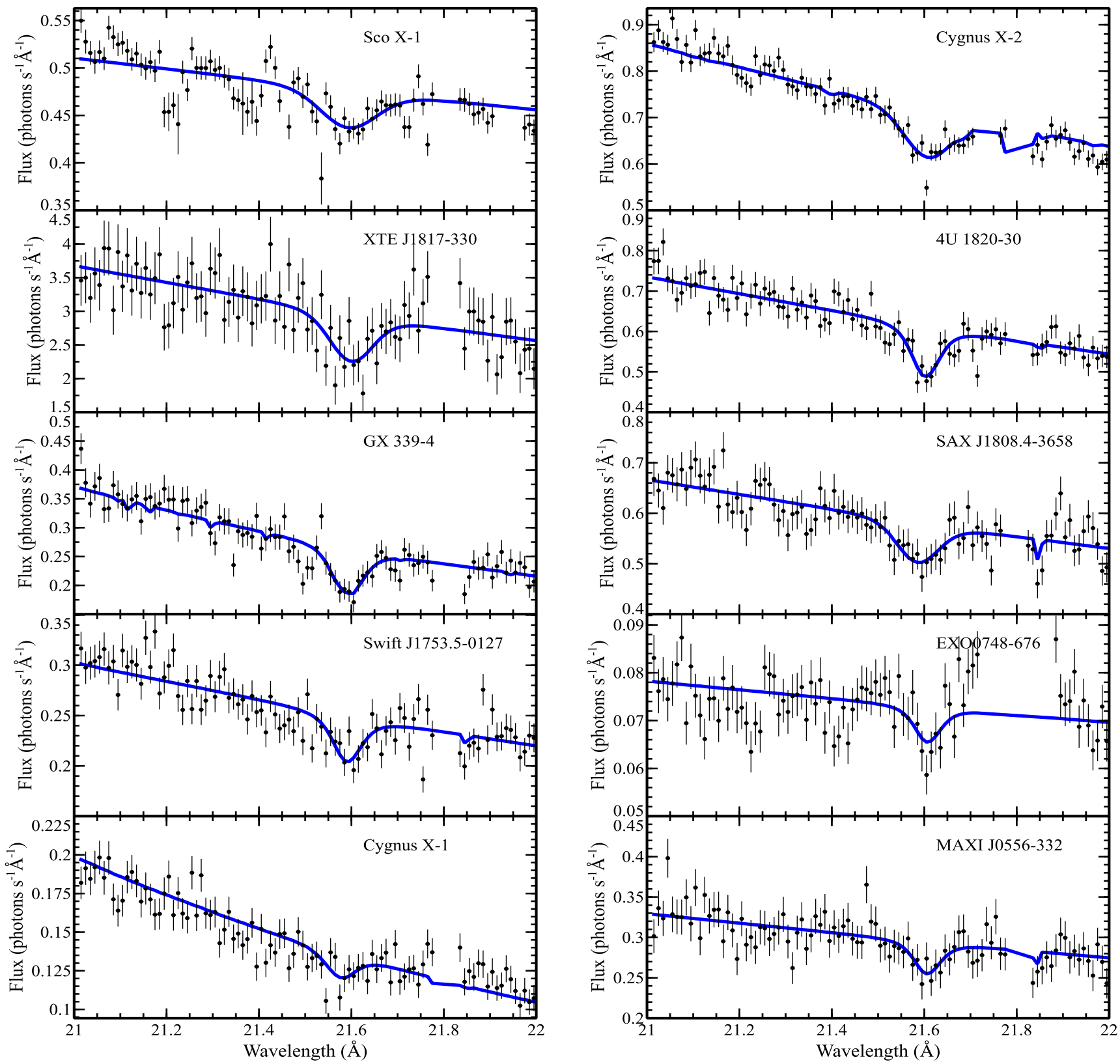

Figure 7. RGS spectra between 21 and $22 \AA$ for each target. The blue line is the fitted continuum. This is only for demonstration purpose because for each target the spectra were jointly fitted with the same absorption line parameters but different continuum level. The structure at $\sim 21.8 \AA$ in each panel is an instrumental feature, and is ignored in the fit.

\section{APPENDIX}

We present all the target spectra in Figure 7-10 in the 21-22 $\AA$ wavelength range. The blue line in each panel is the model fitted continuum and absorption line. The model fitted continuum in these figures are only for display purpose because for each target the continuum changes among different observations. There is a detector feature at about $21.82 \AA$ so we have excluded this region in fitting the spectrum. 

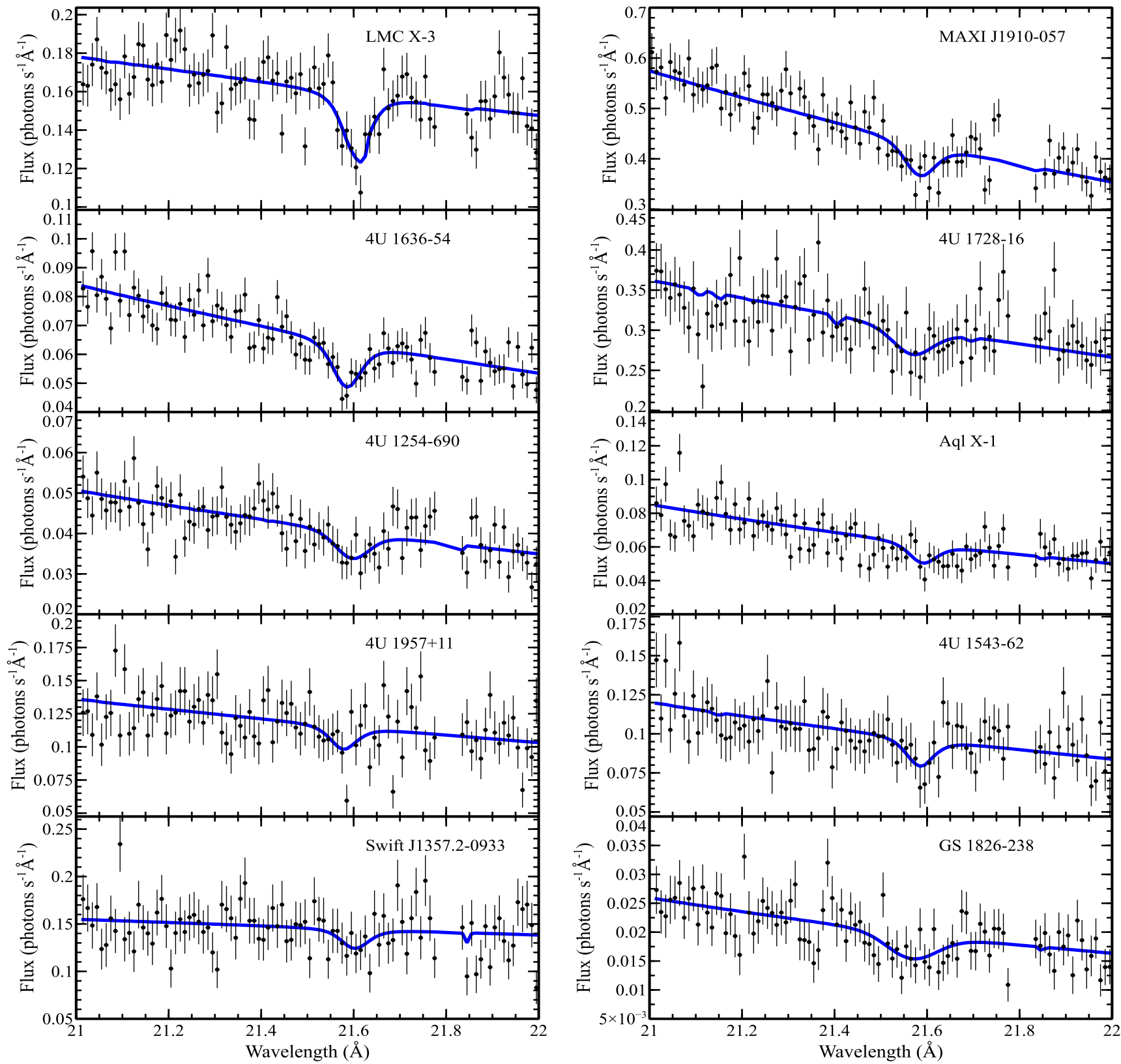

Figure 8. Same as Figure 7 but for different targets. 

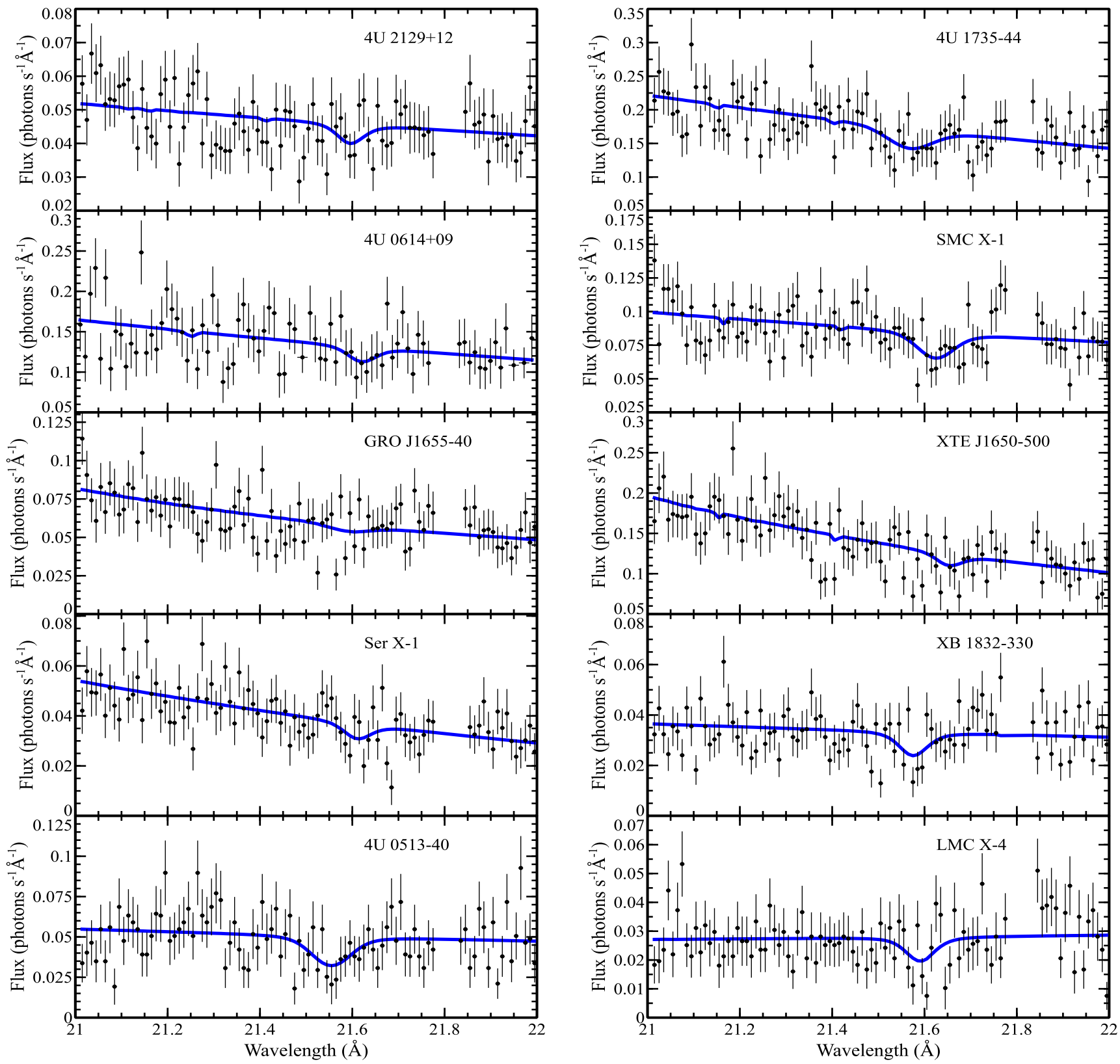

Figure 9. Same as Figure 7 but for different targets. 

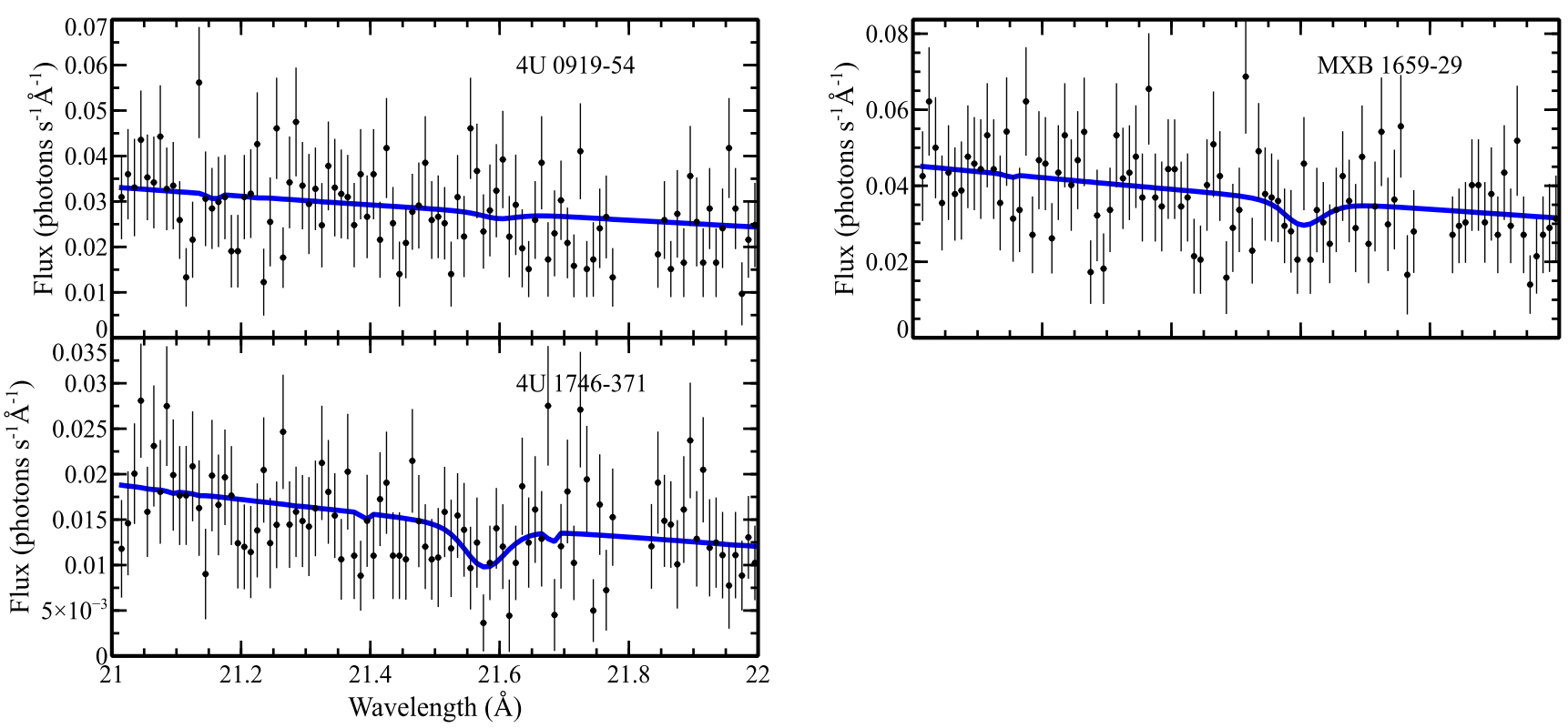

Figure 10. Same as Figure 7 but for different targets. 